Supporting Information

\title{
P-Superdoped Graphene: Synthesis and Magnetic Properties
}

Lihua Lin, ${ }^{\dagger},+$ Lin Fu,${ }^{\dagger}$ Kaiyu Zhang, ${ }^{\dagger}$ Jie Chen, ${ }^{\dagger}$ Weili Zhang, ${ }^{\dagger}$ Shaolong Tang, ${ }^{\dagger}$

Youwei Du, ${ }^{\dagger}$ and Nujiang Tang*,†

†National Laboratory of Solid State Microstructures, Collaborative Innovation Center of

Advanced Microstructures, Jiangsu Provincial Key Laboratory for Nanotechnology, Nanjing

University, Nanjing 210093, China

University Physics Experiment Center, University of Shanghai for Science and Technology,

Shanghai 200093, China

Email address: tangnujiang@nju.edu.cn

\section{This PDF file includes:}

Supporting Figures 


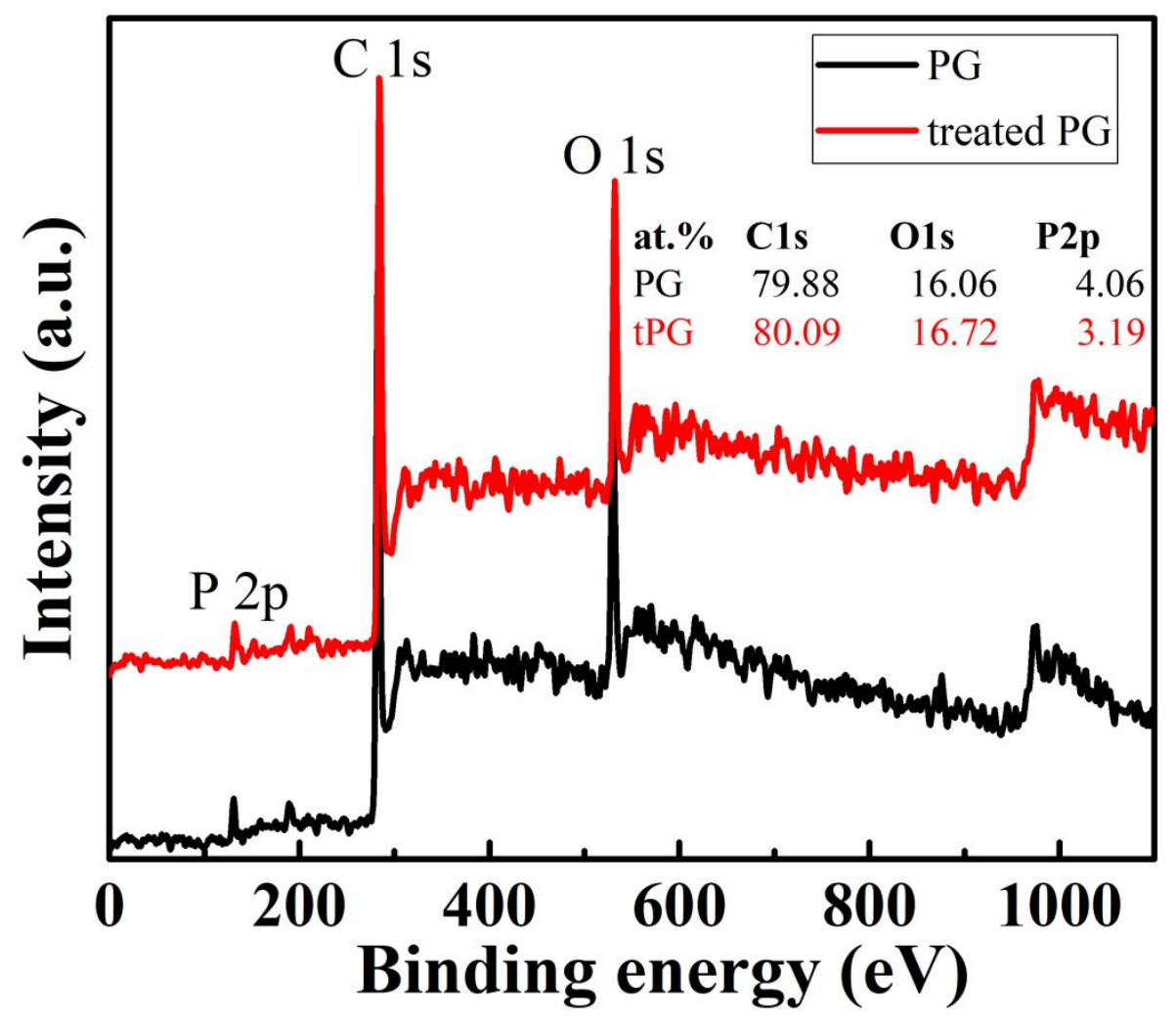

Figure S1. XPS spectra of P-doped graphene (PG) treated with dilute nitric acid solution before and after. There is only a slight change of P content in PG samples before and after treating by dilute nitric acid solution, which is within the range of measurement error, indicating that the $\mathrm{P}$ atom is successfully embedded in the graphene lattice or forms a C-P bond with the $\mathrm{C}$ atom. 

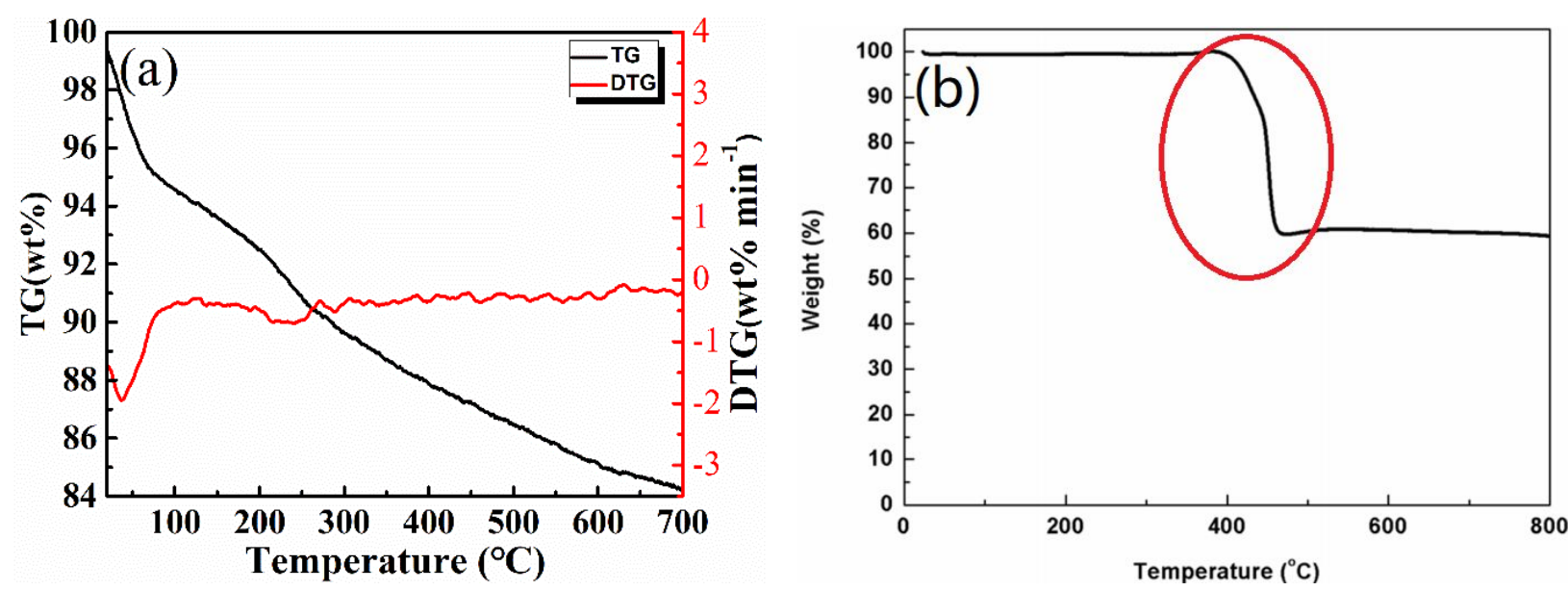

Figure S2. (a) Thermogravimetric analysis (TGA) of PG in argon from room temperature to 700

${ }^{\circ} \mathrm{C}$. (b) TGA of red P-SWCNT composite in argon from room temperature to $800{ }^{\circ} \mathrm{C}$ from ref.(1). ${ }^{1}$ As known, the boiling points of red phosphorus, white phosphorus, and $\mathrm{P}_{2} \mathrm{O}_{5}$ are 416 , 280.5 and $360{ }^{\circ} \mathrm{C}$, respectively. There is no jump in TGA and DTG curves at the range of 200-500 ${ }^{\circ} \mathrm{C}$ in Figure S2(a), indicating that $\mathrm{P}$ atoms are successfully embedded in the graphene lattice forming the P-C bonds, rather than covering the surface of $\mathrm{rFGi}$ in the form of red phosphorus, white phosphorus or $\mathrm{P}_{2} \mathrm{O}_{5}$. However, in Figure S2(b), TGA of red P-SWCNT composite exhibits obvious jump at $400-430 \quad{ }^{\circ} \mathrm{C}$, which corresponds to the sublimation temperature of red phosphorus and its oxides in the ranged from 350 to $420{ }^{\circ} \mathrm{C}$. It confirmed further that the P atoms are successfully doped in the graphene lattice. 

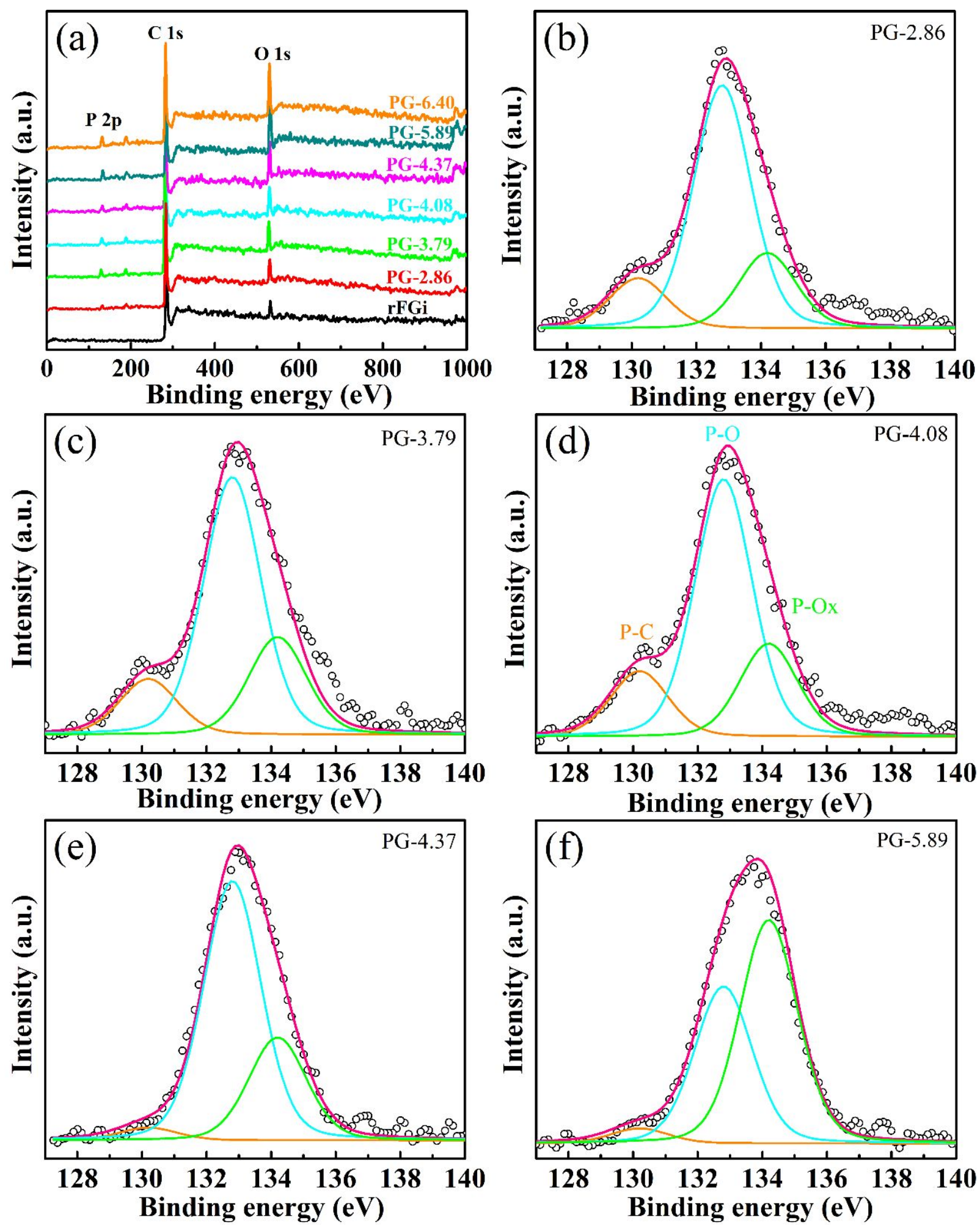

Figure S3. (a) XPS spectra of rFGi and PG samples. High resolution P 2p XPS spectrum of PG-2.86 (b), PG-3.79 (c), PG-4.08 (d), PG-4.37 (e) and PG-5.89 (f). 

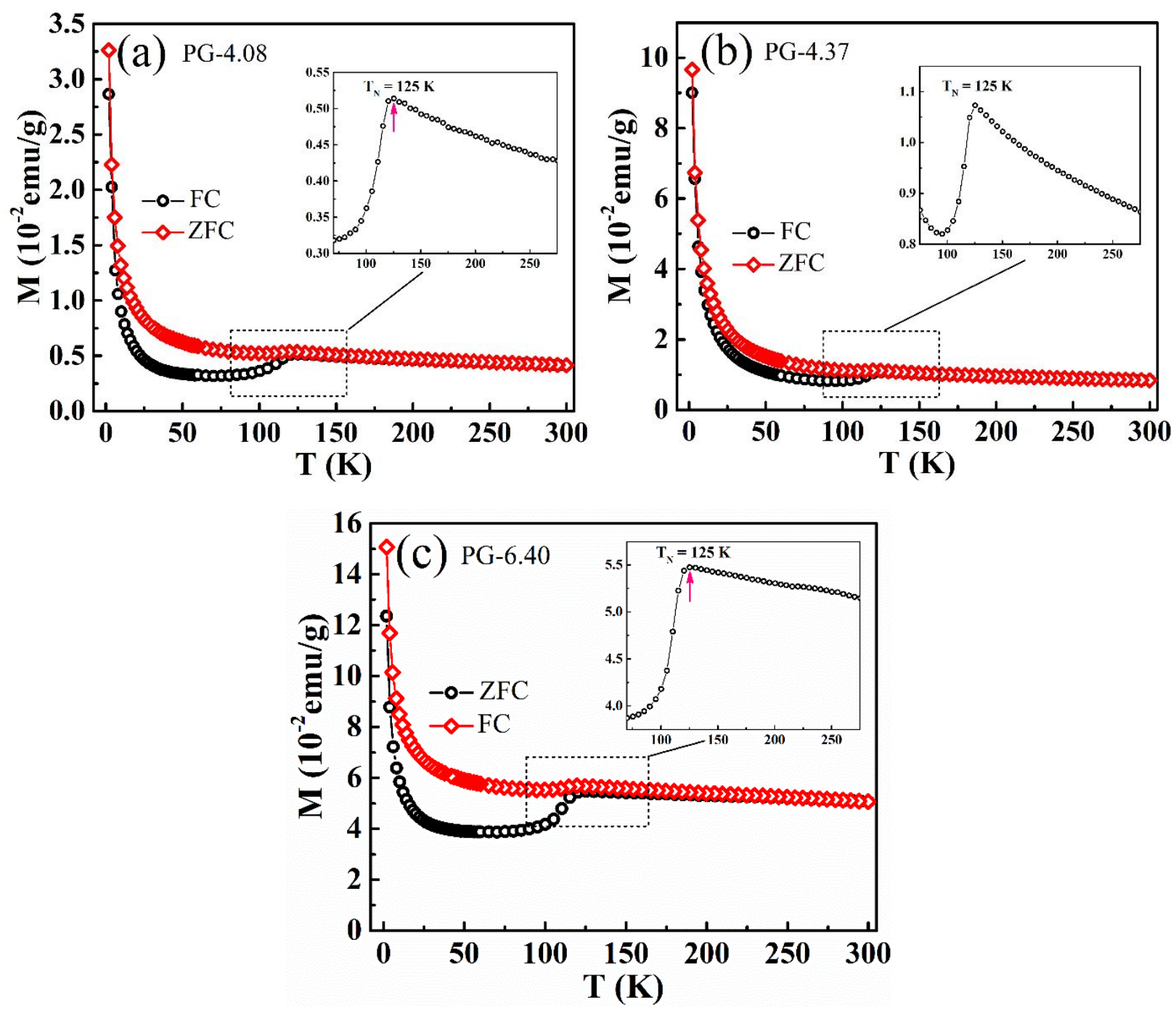

Figure S4. ZFC and FC M-T curves of PG-4.08 (a), PG-4.37 (b) and PG-6.40 (c) measured from 2 to $300 \mathrm{~K}$ under the applied field $H=0.1 \mathrm{kOe}$. The upper right inset is the magnification of the dashed region. 


\section{REFERENCES}

(1) Zhu, Y. J.; Wen, Y.; Fan, X. L.; Gao, T.; Han, F. D.; Luo, C.; Liou, S. C.; Wang, C. S. Red

Phosphorus Single-Walled Carbon Nanotube Composite as a Superior Anode for Sodium Ion

Batteries. ACS Nano 2015, 9, 3254-3264. 